\title{
Análisis de la presencia de las hermandades rocieras en las redes sociales
}

\author{
Analysis of the presence of the Rocío brotherhoods in the social \\ networks
}

\author{
Manuel Jesús Cartes Barroso cartesbarroso@us.es \\ https://orcid.org/0000-0003-1768-275X \\ Universidad de Sevilla (España)
}

\section{Resumen}

Como asociaciones públicas de fieles dentro de la Iglesia católica, las hermandades y cofradías, al igual que cualquier otra institución o colectivo, también tienen la necesidad de 
comunicar y mantener contacto con sus hermanos y seguidores. Hasta ahora han alcanzado estos propósitos a través de diversas publicaciones impresas y, en los últimos años, gracias a las distintas plataformas 2.0. Un buen ejemplo, en este sentido, es el de las hermandades rocieras, que cuentan con un gran número de seguidores y una gran participación en los medios sociales, aunque no siempre de forma acertada ni en base a una estrategia de comunicación. Por ello, este artículo pretende examinar la presencia e intentar comprender el uso de las redes sociales en estas corporaciones religiosas.

Palabras clave: Rocío; redes sociales; web 2.0; comunicación institucional; hermandad.

\section{Abstract}

Fraternities and brotherhoods, as public associations of the faithful within the Catholic Church, also have the need to communicate and maintain contact with members of the brotherhoods and their followers, like any other institution or collective. They have achieved these objectives through various printed publications to date and, furthermore, in recent years thanks to the different 2.0 platforms. In this sense, the Rocío brotherhoods are a good example, having a large number of followers and a great participation in social media, although not always correctly or based on a communication strategy. Therefore, this article aims to examine the presence and try to understand the use of social networks in these religious corporations.

Keywords: Rocío; social networks; web 2.0; institutional communication; brotherhood.

Un claro exponente de la religiosidad popular en España, por la gran universalidad que tiene en la actualidad, es el referente al Rocío. La conocida romería del Rocío, en la provincia de Huelva, es una manifestación religiosa con una gran repercusión en diversos puntos 
geográficos del mundo y, con mayor importancia, en muchas regiones españolas, no sólo en la comunidad autónoma andaluza -en donde tiene lugar su fiesta anual en torno a la solemnidad de Pentecostés-, reuniendo en los últimos tiempos a más de un millón de personas. Igualmente, a lo largo del año tienen lugar otras celebraciones importantes como las peregrinaciones extraordinarias de las hermandades filiales rocieras, así como el denominado Rocío Chico, en el mes de agosto, en el que se conmemora el voto de acción de gracias que la villa de Almonte realizó en 1813 a su patrona, la Virgen del Rocío, por haberle liberado de la sanguinaria amenaza de las tropas francesas durante la invasión napoleónica. Por todo ello, el Rocío y todo lo que le rodea es considerado como un importante movimiento creencial que se ha intensificado notablemente a partir del siglo XX (Padilla Díaz de la Serna, 2007).

La proyección informativa del fenómeno rociero es tal que su difusión, merced al interés de los ciudadanos por todo lo concerniente al Rocío, trasciende más allá de los días de la romería. En la actualidad, la información rociera publicada por los medios de comunicación -habitualmente los de ámbito local- resulta de gran utilidad para conocer y comprender todos los aspectos de esta peregrinación mariana, bien sean particularidades o novedades de la romería, testimonios de fe, advertencias de los operativos de seguridad u otros detalles. Y por otro lado, también reconocemos la existencia de una comunicación institucional rociera a cargo de la Hermandad Matriz de Almonte y sus 121 hermandades filiales, reconocidas como tal a fecha de enero de 2018 (1). A los medios y canales de comunicación propios se han sumado en los últimos años las redes sociales, que han modificado singularmente la manera de comunicar de estas asociaciones religiosas con sus hermanos, devotos y seguidores, como veremos en nuestra investigación.

Síntesis de la relevancia de las redes sociales

A principios del presente siglo, fundamentalmente, hemos asistido a una auténtica revolución de las nuevas tecnologías de la información y la comunicación, que habiendo penetrado soberanamente en la sociedad, han conseguido alterar nuestra forma de vida y nuestras costumbres, al mismo tiempo que la forma de comunicar, ya sea en el contexto familiar, laboral o académico. 
Internet nos ha abierto múltiples posibilidades en este sentido, destacando la relevancia de la World Wide Web (web). Como evolución natural de la web, Tim O'Reilly acuña en 2004 el término web 2.0, con el que, entre otros aspectos, distingue la interacción de los usuarios con respecto a la web 1.0, es decir, comparando el paso de unos sujetos pasivos a unos usuarios participativos (O'Reilly, 2005). Una correcta definición de la web 2.0 es la de Alberto Ortiz de Zárate Tercero:

\begin{abstract}
Un fenómeno social en relación con la creación y distribución de contenidos en Internet, caracterizado por la comunicación abierta, la descentralización de autoridad, la libertad de compartir y usar, dentro de un enfoque que trata a las relaciones humanas y económicas como conversaciones (2008: 18).
\end{abstract}

En relación con esta participación activa del usuario y su poder para crear y transmitir contenidos, se produce a comienzos del siglo XXI un espectacular auge de los social media $o$ medios sociales, destacando entre ellos las redes sociales online. Herrero Curiel (2013: 49) describe que las redes sociales son "nuevos canales de comunicación e información online donde los usuarios de internet se conectan con sus perfiles personales, profesionales o ambos, a través de diferentes dispositivos electrónicos". A día de hoy, millones de personas son usuarios y consumidores de estos canales prácticamente en todo el mundo, existiendo una amplia bibliografía sobre este tema tanto a nivel general (Barnett, 2011; Ryan, 2011; Van Dijck, 2013 -entre otros autores-), como en múltiples ámbitos, siendo una importante línea de investigación para académicos y docentes.

De acuerdo con Fernández (2008), entre las posibilidades que ofrecen las redes sociales están dar visibilidad a nuestra red de contactos, acceder a otros contactos, comunicar y compartir contenido y, especialmente, la creación de comunidades en base a intereses comunes donde intercambiar ideas y establecer relaciones con personas. Además, a estas ventajas hay que añadir que plataformas como Twitter y Facebook se han convertido en fuentes de noticias para miles de personas (Pew Research Center, 2015).

Como venimos diciendo, en el caso de las redes sociales online existe un alto grado de penetración en la sociedad, siendo utilizadas regularmente por aproximadamente un tercio de la población mundial en 2017 (eMarketer, 2018). En España, por ejemplo, el porcentaje de su uso es considerablemente alto, al representar al $85 \%$ de las personas que navegan por internet 
de 16-65 años (IAB, 2018), unos datos que confirman la importancia que tienen en la actualidad las redes sociales.

Y es que la web 2.0 ofrece a las empresas y organizaciones, tanto públicas como privadas, no sólo la oportunidad de comunicar, sino de interactuar con los usuarios, independientemente del momento y lugar en el que estos se encuentren. Así se explica su pujante implantación en las estrategias de comunicación, al ser una forma más cercana para comunicar y, sobre todo, interaccionar con los ciudadanos. La Academia también ha fijado su mirada hasta esta realidad y, prueba de ello, son las investigaciones centradas en la relación de las redes sociales y la comunicación institucional, entre ellas las de Gandolfo (2014) y Cancelo Sanmartín, Rebeil Corella y Gabino Campos (2015).

Hermandades 2.0

Así como compañías e instituciones entendieron hace algunos años que necesitaban comunicar sus acciones, eventos y resultados al público general -no sólo a sus clientes o seguidores-, la Iglesia católica ha actuado de igual forma. En concreto, asociaciones religiosas como las que son objeto de nuestro análisis deben comunicar por varias razones. En primer lugar, porque forman parte de una institución mayor, la Iglesia católica, encargada de divulgar el anuncio evangélico a todos los ciudadanos y "llegar a los confines de la tierra" (2). Por otro lado, porque los miembros de estas corporaciones, los hermanos, requieren estar informados de la vida de la hermandad, siendo así mismo una potente herramienta para formar y evangelizar. Y, finalmente, porque además de los hermanos, otros ciudadanos también están interesados en conocer qué actividades tienen las hermandades, bien a través de los medios de comunicación o directamente en los canales informativos de las mismas (Cartes Barroso, 2016).

En este sentido, no es extraño que publicaciones como revistas, hojas, boletines y, en los últimos tiempos, anuarios, tengan desde hace bastante tiempo un espacio destacado en el quehacer de las hermandades, independientemente de la temporalidad de dichas ediciones. Igualmente, las páginas webs han ampliado la necesidad comunicativa de las cofradías, posibilitando el acceso a un contenido valioso para los hermanos, seguidores y público en 
general. Pero sobre todo, el rol comunicador de las hermandades se ha visto intensificado desde la irrupción de las redes sociales, al sumarse al uso de las plataformas 2.0, con el objetivo de ampliar aún más sus posibilidades comunicativas y, especialmente, de entrar en contacto con sus hermanos y fieles, al mismo tiempo que implantar o reforzar su presencia digital, convirtiéndose en hermandades 2.0.

Pero estas asociaciones, aunque no ocurre con frecuencia, deben seguir una estrategia en este sentido y no lanzarse a la aventura en las redes sociales. Deben planificar qué y cómo van a comunicar, sin olvidar su integración en la Iglesia y a quiénes van dirigidos sus mensajes. Así lo sintetiza José María La Porte:

\footnotetext{
Las instituciones eclesiales desarrollan sus actividades en el mundo y comunican con el mundo, pero no deben adoptar acríticamente cualquier modelo de comunicación, porque no todos, aunque sean eficaces y legítimos en el ámbito comercial, son conformes a la identidad eclesial (2012: 207).
}

Por todo ello puede defenderse la necesidad de comunicación de las hermandades y cofradías, contando para ello con unos medios de comunicación propios y una comunicación propia, especialmente en las plataformas 2.0, aceptando y asumiendo los cambios que se produzcan en el futuro.

\section{Comunicación y Rocío}

En este apartado desarrollamos brevemente la relación entre comunicación y Rocío, partiendo de la idea clave de la existencia de una comunicación institucional rociera, es decir, la que llevan a cabo las distintas hermandades y asociaciones rocieras en diferentes publicaciones, medios y canales informativos, ya sean boletines, revistas, hojas, anuarios, comunicados, circulares, notas de prensa, páginas webs y, en la actualidad, las redes sociales. Por un lado se encuentra la Hermandad Matriz de Almonte, que tiene el papel de ser garante y promotora 
de la devoción hacia Nuestra Señora del Rocío y, por otro, sus hermandades filiales, no filiales y asociaciones. En todos estos casos, el objetivo de estas asociaciones públicas de fieles nacidas dentro de la Iglesia católica es comunicar y difundir su vida interna, promover la interacción y las relaciones públicas con personas, entidades e instituciones y, en definitiva, relacionarse y servir a la sociedad, fundamentalmente a los hermanos y devotos de estas hermandades rocieras.

A pesar de la importancia que tiene el Rocío como fenómeno religioso y social y de su presencia en los medios de comunicación, su vínculo con la comunicación ha sido escasamente estudiado, siendo hasta ahora la obra Periodismo y Rocío (Gómez y Méndez, Méndez Muros y García Estévez, 2015) la única dedicada a esta realidad religiosa desde el punto de vista de la proyección informativa en los medios de comunicación, aunque no desde la perspectiva comunicativa institucional que presenta nuestra investigación. Es por ello que justificamos la necesidad de reflexionar sobre este tema, al entender que es un buen ejemplo de la comunicación organizacional dentro del ámbito religioso.

\section{Objetivos}

El objetivo principal de esta investigación es analizar la presencia y el uso actual de las redes sociales por parte de las hermandades del Rocío, en concreto en sus perfiles de Facebook, Twitter, YouTube e Instagram. Varias de estas hermandades son más que centenarias, mientras que la mayoría se fundaron en el siglo XX, continuando su auge en el presente momento. Es por ello por lo que nos planteamos examinar su penetración en las redes sociales y, además, pretendemos conocer cómo han sido incorporadas y asimiladas por estas asociaciones religiosas con larga historia.

Nuestra indagación, que como argumentamos quiere exclusivamente analizar la presencia de las hermandades rocieras en las redes sociales, comienza en base a una serie de preguntas formuladas al comienzo de la misma, con el propósito de poder dar respuesta a estas cuestiones a su término. Las preguntas planteadas son las siguientes: 
1. ¿Cuál es la presencia de las hermandades rocieras en las redes sociales?

2. ¿Qué redes sociales son las más utilizadas y seguidas?

3. ¿Qué grado de actualización tienen estas plataformas 2.0?

\section{Metodología}

Como metodología hemos llevado a cabo un análisis descriptivo, de enfoque cuantitativo, a partir de los datos existentes en los perfiles de redes sociales de las hermandades rocieras. Hemos decidido examinar las redes sociales YouTube, Facebook, Instagram y Twitter porque son las más utilizadas en nuestro país, con unos porcentajes del $73 \%, 69 \%, 40 \%$ y $39 \%$, respectivamente, superados sólo por WhatsApp, aunque la consideramos más bien como aplicación (We Are Social y Hootsuite, 2018).

Por otro lado, no nos olvidamos de que algunas hermandades rocieras tienen presencia en otras redes sociales como Google+, Pinterest, Tuenti o Flickr, aunque con un uso menor que los medios sociales analizados.

Nuestra investigación se ha centrado en el análisis de la presencia en redes sociales de la Hermandad Matriz de Almonte y sus 121 hermandades filiales, repartidas por todo el territorio español y también en Europa, como es el caso de la Hermandad de Bruselas. La información sobre las mismas se ha obtenido de la página web de la Hermandad Matriz de Almonte (www.hermandadmatrizrocio.org) y a través de la web especializada Rocio.com (www.rocio.com), donde se detallan las hermandades filiales por orden de antigüedad, actualizando el listado existente en cuanto se refiere a las direcciones cibernéticas de estas asociaciones. Este dato de la modernización de las webs de las hermandades filiales es importante, al creer a priori que en sus sitios webs suelen aparecer, generalmente, sus perfiles en las redes sociales. El resultado de nuestra relación puede observarse en la siguiente tabla: 


\begin{tabular}{|c|c|c|}
\hline №. & Hermandad & Web \\
\hline 0 & Almonte & www.hermandadmatrizrocio.org \\
\hline 1 & Villamanrique de la Condesa & www.hermandaddelrociodevillamanrique.com \\
\hline 2 & Pilas & www.hdadrociopilas.org \\
\hline 3 & La Palma del Condado & www.hermandadrociolapalma.blogspot.com \\
\hline 4 & Moguer & www.hdadrociomoguer.com \\
\hline 5 & Sanlúcar de Barrameda & www.hermandaddelrociodesanlucardebarrameda.com \\
\hline 6 & Triana & www.hermandadrociodetriana.org \\
\hline 7 & Umbrete & No \\
\hline 8 & Coria del Río & www.hermandaddecoria.wixsite.com/rocio \\
\hline 9 & Huelva & www.hermandaddelrociodehuelva.com \\
\hline 10 & San Juan del Puerto & www.hermandadrociosanjuan.blogspot.com * \\
\hline 11 & Rociana del Condado & www.hermandadrociorociana.es \\
\hline 12 & Carrión de los Céspedes & www.hermandaddelrociodecarrion.es \\
\hline 13 & Benacazón & www.rociodebenacazon.blogspot.com * \\
\hline 14 & Trigueros & www.hdadrociotrigueros.blogspot.com * \\
\hline 15 & Gines & www.hermandadrociodegines.blogspot.com \\
\hline 16 & Jerez de la Frontera & www.rociojerez.com \\
\hline 17 & Dos Hermanas & www.rociodoshermanas.es \\
\hline 18 & Olivares & www.hermandaddelrociodeolivares.com \\
\hline 19 & Hinojos & No \\
\hline 20 & Bonares & No \\
\hline 21 & La Puebla del Río & www.hdadrociopuebladelrio.es \\
\hline 22 & Bollullos Par del Condado & www.hermandaddelrociobollullos.com \\
\hline 23 & Valverde del Camino & www.hermandaddelrociodevalverdedelcamino.blogspot.com * \\
\hline 24 & Gibraleón & www.hermandadrociogibraleon.com * \\
\hline
\end{tabular}




\begin{tabular}{|c|c|c|}
\hline 25 & Espartinas & www.hermandadrocioespartinas.com \\
\hline 26 & Sanlúcar la Mayor & www.hermandadrociosanlucarlamayor.wordpress.com * \\
\hline 27 & Lucena del Puerto & No \\
\hline 28 & Bollullos de la Mitación & No \\
\hline 29 & Sevilla & www.hermandaddelrociodesevilla.org * \\
\hline 30 & Huévar del Aljarafe & No \\
\hline 31 & Aznalcázar & www.hermandaddelrocioaznalcazar.es \\
\hline 32 & El Puerto de Santa María & No \\
\hline 33 & Madrid & www.hermandaddemadrid.com \\
\hline 34 & Punta Umbría & www.hermandaddelrociodepuntaumbria.es \\
\hline 35 & Puerto Real & No \\
\hline 36 & Barcelona & No \\
\hline 37 & Palos de la Frontera & www.hermandadrociopalos.com * \\
\hline 38 & Emigrantes & www.hermandademigrantesdelrocio.org * \\
\hline 39 & Paterna del Campo & www.hermandadrociopaterna.com \\
\hline 40 & Villanueva del Ariscal & www.hermandadrociovillanuevadelariscal.org \\
\hline 41 & Lucena & www.hermandadrociodelucena.com * \\
\hline 42 & Los Palacios y Villafranca & www.rociolospalacios.com \\
\hline 43 & Écija & No \\
\hline 44 & Villarrasa & No \\
\hline 45 & Isla Cristina & www.hdaddelrociodeislacristina.blogspot.com \\
\hline 46 & Bormujos & www.hermandadrociobormujos.blogspot.com * \\
\hline 47 & Camas & www.hermandadrociocamas.blogspot.com * \\
\hline 48 & Las Palmas de Gran Canaria & www.hdaddelrociolaspalmasdegrancanaria.blogspot.com * \\
\hline 49 & Lebrija & www.hdadrociolebrija.org * \\
\hline 50 & La Línea de la Concepción & No \\
\hline
\end{tabular}




\begin{tabular}{|c|c|c|}
\hline 51 & Córdoba & No \\
\hline 52 & Rota & No \\
\hline 53 & Ayamonte & www.ayamonterociera.blogspot.com * \\
\hline 54 & Villalba del Alcor & No \\
\hline 55 & Granada & www.rociogranada.org \\
\hline 56 & Isla Mayor & www.hermandadislamayor.wordpress.com * \\
\hline 57 & Cabra & No \\
\hline 58 & Málaga & www.realhdaddelrociodemalaga.org \\
\hline 59 & Cádiz & www.hermandaddelrociodecadiz.blogspot.com * \\
\hline 60 & Puente Genil & No \\
\hline 61 & Jaén & www.rociojaen.es \\
\hline 62 & Castillo de Locubín & No \\
\hline 63 & Alcalá de Guadaira & No \\
\hline 64 & Algeciras & No \\
\hline 65 & Marbella & www.hermandaddelrociomarbella.com * \\
\hline 66 & Tocina & www.rociodetocina.blogspot.com * \\
\hline 67 & Gelves & www.rociodegelves.com * \\
\hline 68 & Utrera & No \\
\hline 69 & Almería & www.hermandadrocioalmeria.com * \\
\hline 70 & Cerro del Águila & www.rociodelcerro.com \\
\hline 71 & Sevilla Sur & www.sevillasur.es \\
\hline 72 & Toledo & www.hermandaddelrociodetoledo.blogspot.com * \\
\hline 73 & Almensilla & www.hermandadrociodealmensilla.blogspot.com * \\
\hline 74 & Las Cabezas de San Juan & No \\
\hline 75 & San Juan de Aznalfarache & www.rociodesanjuan.blogspot.com * \\
\hline 76 & Fuengirola & www.hermandaddelrociodefuengirola.com/joomla * \\
\hline
\end{tabular}




\begin{tabular}{|c|c|c|}
\hline 77 & Ceuta & www.rocioceuta.es/web \\
\hline 78 & Osuna & www.actiweb.es/hermandadrocioosuna * \\
\hline 79 & Santiponce & www.hermandaddelrociodesantiponce.blogspot.com * \\
\hline 80 & Valencia & www.hermandadrociovalencia.com * \\
\hline 81 & Mairena del Alcor & No \\
\hline 82 & Carmona & www.hermandaddelrociodecarmona.blogspot.com * \\
\hline 83 & Sevilla-Macarena & www.hermandaddelrociodelamacarena.org \\
\hline 84 & Niebla & No \\
\hline 85 & Málaga-La Caleta & www.rociomalagalacaleta.es \\
\hline 86 & Priego de Córdoba & No \\
\hline 87 & Alcalá la Real & www.hermandaddelrocioalcalalareal.blogspot.com * \\
\hline 88 & Ronda & www.rocioronda.com \\
\hline 89 & Badajoz & No \\
\hline 90 & Chucena & No \\
\hline 91 & La Algaba & www.laalgabarociera.blogspot.com \\
\hline 92 & Murcia & No \\
\hline 93 & Manzanilla & No \\
\hline 94 & Tomares & www.hermandaddelrociodetomares.com * \\
\hline 95 & Arcos de la Frontera & No \\
\hline 96 & Bruselas & www.rociobruselas.blogspot.com \\
\hline 97 & Estepona & No \\
\hline 98 & Escacena del Campo & No \\
\hline 99 & Alicante & No \\
\hline 100 & Alcalá de Henares & No \\
\hline 101 & Guadix & www.hermandadrocioguadix.blogspot.com * \\
\hline 102 & Palma de Mallorca & www.hdadrociomallorca.blogspot.com * \\
\hline
\end{tabular}




\begin{tabular}{|c|c|c|}
\hline 103 & Mairena del Aljarafe & www.hermandaddelrociodemairenadelaljarafe.es * \\
\hline 104 & San Fernando & www.hermandadrociosanfernando.com * \\
\hline 105 & Palomares del Río & www.hdadrociopalomaresdelrio.wordpress.com \\
\hline 106 & Morón de la Frontera & No \\
\hline 107 & Pozuelo de Alarcón & www.hdrociopozuelo.info \\
\hline 108 & San Antonio de Portmany & www.hdadrocierasanantonioibiza.com \\
\hline 109 & Cornellá & www.hermandadrociocornella.com \\
\hline 110 & Chiclana de la Frontera & No \\
\hline 111 & Chipiona & $\underline{\text { No }}$ \\
\hline 112 & Castrense & www.hermandadcastrensedelrocio.es * \\
\hline 113 & Albaida del Aljarafe & No \\
\hline 114 & Santa Fe & No \\
\hline 115 & Torrejón de Ardoz & www.torrejonrociero.tk * \\
\hline 116 & Salteras & www.hermandad-ntra-sra-del-rocio-de-salteras.webnode.es \\
\hline 117 & Valencina de la Concepción & www.rociodevalencina.blogspot.com \\
\hline 118 & Cartaya & www.hdaddelrocio-cartaya.blogspot.com * \\
\hline 119 & La Carolina & www.rociolacarolina.blogspot.com * \\
\hline 120 & San Sebastián de los Reyes & www.hermandaddelrociossreyes.com * \\
\hline 121 & El Viso del Alcor & No \\
\hline
\end{tabular}

Tabla 1. Relación de las hermandades rocieras (Matriz y filiales) y sus sitios webs. Fuente: elaboración propia a partir de Hermandad Matriz de Almonte y Rocio.com. * Webs no actualizadas, en mantenimiento o con problemas para acceder.

En otro orden de cosas, en todas las redes sociales analizamos el número de seguidores o amigos, mientras que en el caso de Twitter también se examinan el número de tuits, las listas, el número de seguidos y de 'Me gustas'; en YouTube el número de vídeos y, finalmente, en 
Instagram el número de publicaciones y de seguidos. Los datos se recogieron durante los días 3 y 4 de octubre de 2018, por lo que han podido sufrir algunas variaciones.

Resultados

En la tabla 2 se muestra, a modo general, la presencia de las hermandades filiales rocieras en las redes sociales analizadas, ordenadas aquellas por orden de antigüedad y encabezadas por la Hermandad Matriz. Como examinamos a continuación, también se recogen todas las personas que siguen estas cuentas, ya sean seguidores, suscriptores, amigos, miembros o 'Me gusta'.

\begin{tabular}{|l|c|c|c|c|}
\multicolumn{1}{|c|}{ Hermandad } & Facebook & Twitter & YouTube & Instagram \\
\hline Matriz de Almonte & Me gusta & Seguidores & Suscriptores & Seguidores \\
\hline Villamanrique de la Condesa & 34.710 & 20.669 & 663 & - \\
\hline Pilas & $\begin{array}{c}38.476 \\
\text { amigos }\end{array}$ & - & $5^{*}$ & $1^{*}$ \\
\hline La Palma del Condado & $\begin{array}{c}961 / 2.704 \\
\text { amigos }\end{array}$ & 1.093 & 11 & - \\
\hline Moguer & 7.194 & 2.109 & $211^{*}$ & $1.233^{*}$ \\
\hline Sanlúcar de Barrameda & 3.087 & 690 & - & - \\
\hline Triana & 13.156 & 14.149 & 172 & 1.528 \\
\hline Umbrete & 1.380 & 197 & - & $191^{*}$ \\
\hline Coria del Río & 6.158 & 3.189 & 16 & 1.061 \\
\hline Huelva & 23.260 & 1.035 & 179 & 7.088 \\
\hline
\end{tabular}




\begin{tabular}{|c|c|c|c|c|}
\hline San Juan del Puerto & 1.269 & - & - & - \\
\hline Rociana del Condado & 888 & 1.096 * & - & - \\
\hline Carrión de los Céspedes & 1.245 & 57 & - & - \\
\hline Benacazón & 4.088 & 78 & - & 516 \\
\hline Trigueros & 4.603 & 759 & - & - \\
\hline Gines & 8.130 & 3.352 & 59 & 1.395 \\
\hline Jerez de la Frontera & 3.281 & - & - & - \\
\hline Dos Hermanas & 6.578 & 3.404 & - & - \\
\hline Olivares & 2.441 & - & 0 * & - \\
\hline Hinojos & 1.823 & 20 & - & 292 \\
\hline Bonares & 2.291 & 417 & - & - \\
\hline La Puebla del Río & 533 & 2.147 & 21 & - \\
\hline Bollullos Par del Condado & 1.297 & - & 4 * & - \\
\hline Valverde del Camino & 982 & - & 11 * & - \\
\hline Gibraleón & 830 & 879 * & - & - \\
\hline Espartinas & 1.988 & 2.391 & 24 * & - \\
\hline Sanlúcar la Mayor & $\begin{array}{l}1.387^{*} / \mathrm{No} \\
\text { disponible }\end{array}$ & 1.493 * & 33 * & - \\
\hline Lucena del Puerto & 1.790 & - & - & 104 \\
\hline Bollullos de la Mitación & 1.373 & $102 *$ & - & - \\
\hline Sevilla & 4.409 & 2.408 & - & - \\
\hline Huévar del Aljarafe & 1.384 & 319 * & - & - \\
\hline Aznalcázar & 433 & 906 & - & - \\
\hline El Puerto de Santa María & 414 & - & - & - \\
\hline Madrid & 1.723 miembros & $860 *$ & - & 93 * \\
\hline Punta Umbría & 717 & 52 & 4 & 485 \\
\hline
\end{tabular}




\begin{tabular}{|c|c|c|c|c|}
\hline Puerto Real & 450 miembros & - & - & 384 \\
\hline Barcelona & 4.139 & - & - & - \\
\hline Palos de la Frontera & 1.557 & - & $7 *$ & - \\
\hline Emigrantes & 8.699 & 382 * & - & 830 \\
\hline Paterna del Campo & 425 miembros & - & - & - \\
\hline Villanueva del Ariscal & 1.499 & 1.948 & 16 * & - \\
\hline Lucena & 1.145 & 1.190 & $2 *$ & 670 \\
\hline Los Palacios y Villafranca & 2.581 & 388 & $6 *$ & 562 \\
\hline Écija & 2.570 & 965 & - & 915 \\
\hline Villarrasa & 402 & - & - & - \\
\hline Isla Cristina & No disponible & - & - & - \\
\hline Bormujos & $\begin{array}{c}3.913 / 749 \\
\text { amigos }\end{array}$ & 1.035 & 2 & - \\
\hline Camas & $365 / 492$ amigos & 637 & - & - \\
\hline Las Palmas de Gran Canaria & 3.156 & - & - & - \\
\hline Lebrija & 1.758 & 304 * & $0 *$ & 158 * \\
\hline La Línea de la Concepción & 1.328 miembros & - & - & - \\
\hline Córdoba & 11.549 & 3.212 & - & - \\
\hline Rota & 1.073 & 511 * & - & - \\
\hline Ayamonte & 2.979 & 679 * & - & - \\
\hline Villalba del Alcor & 3.185 amigos & 466 * & - & - \\
\hline Granada & $\begin{array}{c}\text { 338/No } \\
\text { disponible }\end{array}$ & 999 & - & - \\
\hline Isla Mayor & No disponible & 866 * & - & - \\
\hline Cabra & 1.483 & 388 * & - & 201 \\
\hline Málaga & 4.852 & 4.696 & - & - \\
\hline Cádiz & 1.221 & 609 & - & - \\
\hline
\end{tabular}




\begin{tabular}{|c|c|c|c|c|}
\hline Puente Genil & 1.501 & $582 *$ & - & - \\
\hline Jaén & 3.175 & - & - & 323 \\
\hline Castillo de Locubín & 1.551 & - & - & - \\
\hline Alcalá de Guadaira & 1.942 & - & 0 * & - \\
\hline Algeciras & 2.433 & - & - & - \\
\hline Marbella & 2.078 & 823 * & - & - \\
\hline Tocina & 590 & 114 & - & 110 \\
\hline Gelves & 2.795 & - & - & - \\
\hline Utrera & 609 & 273 & - & 589 \\
\hline Almería & 6.642 & 2.261 & - & 546 \\
\hline Cerro del Águila & 1.218 & 3.554 & 30 & - \\
\hline Sevilla Sur & 2.212 & 3.537 & 4 * & 876 \\
\hline Toledo & 3.823 & 219 * & - & - \\
\hline Almensilla & 2.969 amigos & - & $7 *$ & - \\
\hline Las Cabezas de San Juan & 2.161 & 2.100 & 16 * & - \\
\hline San Juan de Aznalfarache & 2.668 & - & - & - \\
\hline Fuengirola & 5.235 & 321 & 29 & 326 \\
\hline Ceuta & $\begin{array}{c}474 \text { */2.992 } \\
\text { amigos }\end{array}$ & - & 1 * & 981 \\
\hline Osuna & 678 & - & - & 175 \\
\hline Santiponce & $\begin{array}{c}1.713 / 2.139 \\
\text { amigos }\end{array}$ & 426 & - & 819 \\
\hline Valencia & 4.971 & $625^{*}$ & 12 & - \\
\hline Mairena del Alcor & 868 & $404 *$ & 3 * & - \\
\hline Carmona & $\begin{array}{c}1.364 / 2.014 \\
\text { amigos }\end{array}$ & 545 & - & - \\
\hline Sevilla-Macarena & 4.138 & 2.845 & - & 1.088 \\
\hline
\end{tabular}




\begin{tabular}{|c|c|c|c|c|}
\hline Niebla & 2.180 amigos & - & - & - \\
\hline Málaga-La Caleta & 2.861 & 2.753 & 49 & 738 \\
\hline Priego de Córdoba & 959 & - & - & - \\
\hline Alcalá la Real & 523 & - & - & 138 \\
\hline Ronda & 2.218 & 1.975 & - & - \\
\hline Badajoz & No disponible & - & - & - \\
\hline Chucena & 2.605 & 305 * & - & 1.091 \\
\hline La Algaba & $\begin{array}{c}2.023 / 2.354 \\
\text { amigos }\end{array}$ & 1.421 & - & 728 \\
\hline Murcia & 5.832 & 612 * & $2 *$ & - \\
\hline Manzanilla & 90 miembros & - & - & - \\
\hline Tomares & 1.379 & 803 & - & 475 \\
\hline Arcos de la Frontera & 1.487 & 694 * & - & 682 \\
\hline Bruselas & 4.503 & - & - & - \\
\hline Estepona & 6.358 & 861 & - & 1.103 \\
\hline Escacena del Campo & $\begin{array}{c}130 * / 1.225 \\
\text { amigos }\end{array}$ & - & - & - \\
\hline Alicante & 2.443 & 1.140 * & - & - \\
\hline Alcalá de Henares & $\begin{array}{c}994 / 2.488 \\
\text { amigos }\end{array}$ & - & 2 & - \\
\hline Guadix & 1.140 & - & 16 * & - \\
\hline Palma de Mallorca & 4.448 & - & - & - \\
\hline Mairena del Aljarafe & 1.673 & 954 & - & 171 \\
\hline San Fernando & 1.304 & 448 & - & 684 \\
\hline Palomares del Río & 3.109 & - & 12 & 231 \\
\hline Morón de la Frontera & 1.555 & 1.903 & - & 715 \\
\hline Pozuelo de Alarcón & 951 & 570 & 17 & - \\
\hline
\end{tabular}




\begin{tabular}{|c|c|c|c|c|}
\hline San Antonio de Portmany & 671 & - & - & 187 \\
\hline Cornellá & 5.038 & 1.153 & - & - \\
\hline Chiclana de la Frontera & 8.104 & - & - & - \\
\hline Chipiona & 6.146 & 865 & - & 104 * \\
\hline Castrense & 2.050 & 823 & - & 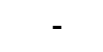 \\
\hline Albaida del Aljarafe & 1.629 & 2.068 & - & - \\
\hline Santa Fe & 1.037 & 218 & - & 827 * \\
\hline Torrejón de Ardoz & 2.431 & 1.880 & $3 *$ & 547 \\
\hline Salteras & 2.337 & 1.579 & 16 * & - \\
\hline Valencina de la Concepción & 2.142 & 1.556 & - & - \\
\hline Cartaya & 1.977 & - & - & - \\
\hline La Carolina & 996 & - & 11 * & - \\
\hline San Sebastián de los Reyes & 2.171 & - & - & - \\
\hline El Viso del Alcor & 2.014 & 589 & 13 & 1.006 \\
\hline
\end{tabular}

Tabla 2. Presencia y número de seguidores de las hermandades rocieras en las principales redes sociales online. Fuente: elaboración propia. *Cuenta o perfil sin actualización desde antes de la romería del Rocío de 2017.

Gracias a los resultados de esta tabla podemos apreciar, en primer lugar, la presencia de las hermandades rocieras en las redes sociales online. Todas las filiales disponen de perfiles, por lo menos, en alguna red social. La red social más utilizada es Facebook, con diferencia, seguida de Twitter, Instagram y YouTube. Concretamente, el 100\% de las hermandades utiliza Facebook, el 66,4\% tiene una cuenta en Twitter (81 hermandades), el 37,7\% usa Instagram (46 hermandades) y, finalmente, el $32,8 \%$ tiene un canal en YouTube (40 hermandades).

Por otro lado, en contra de lo que suponíamos, estas redes sociales suelen aparecer con poca frecuencia en las páginas webs de sus respectivas hermandades. En este sentido, en la mayoría de las ocasiones hemos tenido que buscar las cuentas personalmente en cada una de las redes sociales analizadas o bien en buscadores de Internet, teniendo algunos de estos perfiles una actualización nula o bien no se usan en la actualidad. Un caso llamativo es el de 
Facebook, donde encontramos tanto páginas (fan pages) como perfiles personales para agregar amigos -dos de ellos con nombre de persona (Pilas y Ceuta)-, además de grupos públicos (Madrid, Puerto Real y Manzanilla) o privados (Paterna del Campo y La Línea de la Concepción) bajo la denominación de una hermandad rociera. Incluso hay 12 hermandades (Pilas, La Palma del Condado, Sanlúcar la Mayor, Bormujos, Camas, Granada, Ceuta, Santiponce, Carmona, La Algaba, Escacena del Campo y Alcalá de Henares) que tienen tanto páginas como perfiles personales -con uso o sin uso-, y otras 6 exclusivamente perfiles personales (Isla Cristina, Villalba del Alcor, Isla Mayor, Almensilla, Niebla y Badajoz), a pesar de que la normativa de la red social de Mark Zuckerberg prohíben el uso de los perfiles personales para empresas y asociaciones, como es el caso.

Y otro problema hallado en nuestro análisis, tanto en Facebook como en Twitter, es el elevado número de cuentas que tienen algunas hermandades rocieras en estas redes sociales, en ocasiones dos o tres distintas. Creemos que la creación de estas cuentas puede deberse a la existencia e incorporación de varias juntas de gobierno de hermandad, que quizá dejen inactivas antiguas cuentas y abran a su llegada nuevos perfiles. Consideramos que, ajenos a los motivos que sean, se trata de una mala práctica y, sobre todo, una lacra en la labor comunicativa de las hermandades, por lo que sería útil eliminar las cuentas sin uso y continuar con las existentes en el futuro, donde se indique que éstas son de carácter oficial. Lo mismo deberían hacer con las webs y los blogs obsoletos.

Por otro lado, en esta tabla aparecen las personas que siguen estos perfiles de redes sociales siendo, evidentemente, muy acusadas las diferencias en algunos casos. En líneas generales, las hermandades rocieras con más hermanos y más conocidas popularmente son las que tienen más seguidores, como pueden ser la Hermandad Matriz de Almonte y las filiales de Huelva, Emigrantes, Triana, Gines, Moguer y Córdoba, por ejemplo. Facebook es la red social que cuenta con más seguidores en las hermandades del Rocío, seguida de Twitter e Instagram, mientras que los suscriptores de YouTube son poco relevantes.

En este sentido, las hermandades con más seguidores en la red social de Mark Zuckerberg son las de Almonte (34.710 seguidores), Huelva (23.260 seguidores), Triana (13.156 seguidores) y Córdoba (11.549 seguidores), todas ellas con más de 10.000 seguidores. A continuación, veremos cuál es la presencia de las filiales rocieras en las restantes redes sociales. 


\begin{tabular}{|c|c|c|c|c|}
\hline Usuario & Tuits & Me Gusta & Seguidos & Listas \\
\hline $\begin{array}{l}\text { Hermandad Matriz de Almonte } \\
\text { (@hdadmatrizrocio) }\end{array}$ & 4.082 & 369 & 505 & 6 \\
\hline Pilas (@HDADROCIOPILAS) & 489 & 55 & 82 & 1 \\
\hline La Palma del Condado (@RocioLaPalma) & 1.424 & 96 & 324 & - \\
\hline Moguer (@HdadRocioMoguer) & 735 & 148 & 1.089 & - \\
\hline Sanlúcar de Barrameda (@rociodesanlucar) & 164 & 12 & 470 & - \\
\hline Triana (@rociodetriana) & 2.893 & 858 & 208 & - \\
\hline Umbrete (@rociodeumbrete) & 72 & 2 & 80 & - \\
\hline Coria del Río (@hdadrociocoria) & 1.180 & 231 & 196 & - \\
\hline Huelva (@hdadRocioHuelva) & 779 & 478 & 197 & - \\
\hline Rociana del Condado (@HdaRocioRocian) & 181 & 4 & 408 & 2 \\
\hline Carrión de los Céspedes (@HRociodeCarrion) & 57 & 36 & 83 & - \\
\hline Benacazón (@HdadBenacazon) & 15 & 4 & 84 & - \\
\hline Trigueros (@HdadTrigueros) & 253 & 4 & 126 & - \\
\hline Gines (@ROCIODEGINES) & 912 & 175 & 189 & - \\
\hline Dos Hermanas (@HdadRocioDosHna) & 2.209 & 118 & 104 & - \\
\hline Hinojos (@rociodehinojos) & 236 & 22 & 10 & - \\
\hline Bonares (@Hdad_Bonares) & 154 & 17 & 180 & - \\
\hline La Puebla del Río (@HdadRocioPuebla) & 562 & 21 & 51 & - \\
\hline Gibraleón (@hdadrociogibra) & 285 & 62 & 545 & - \\
\hline Espartinas (@RocioEspartinas) & 1.805 & 457 & 81 & - \\
\hline Sanlúcar la Mayor (@HdadRocioSLM) & 929 & 188 & 334 & - \\
\hline Bollullos de la Mitación (@HdadRocioBM) & 9 & - & 55 & - \\
\hline Sevilla (@HdadRociodeSev) & 837 & 652 & 247 & - \\
\hline Huévar del Aljarafe (@RocioHuevar) & 88 & 11 & 116 & - \\
\hline
\end{tabular}




\begin{tabular}{|c|c|c|c|c|}
\hline Aznalcázar (@RocioAznalcazar) & 1.108 & 413 & 110 & - \\
\hline Madrid (@HermandadMadrid) & 6 & - & 59 & - \\
\hline Punta Umbría (@hdadrociopunta) & 5 & 2 & 128 & 1 \\
\hline Emigrantes (@EmigrantesRocio) & 281 & - & 134 & - \\
\hline Villanueva del Ariscal (@HdadRocioVvaA) & 1.897 & 2.187 & 192 & - \\
\hline Lucena (@HdadRocioLucena) & 567 & 64 & 729 & - \\
\hline Los Palacios y Villafranca (@rociolspalacios) & 214 & 48 & 197 & - \\
\hline Écija (@HdadRocioEcija) & 1.675 & 343 & 170 & - \\
\hline Bormujos (@HDADBORMUJOS) & 566 & 7 & 248 & - \\
\hline Camas (@HdadRocioCamas) & 216 & 128 & 215 & - \\
\hline Lebrija (@EIRocioLEBRIJA) & 280 & 43 & 136 & - \\
\hline Córdoba (@hdadrociocordob) & 1.753 & 39 & 245 & - \\
\hline Rota (@HdadRocioRota) & 18 & - & 184 & - \\
\hline Ayamonte (@AyamonteRociera) & 25 & - & 146 & - \\
\hline Villalba del Alcor (@hdadrvillalba) & 165 & 81 & 189 & - \\
\hline Granada (@hdadrociogr) & 568 & 215 & 984 & - \\
\hline Isla Mayor (@HddRociolslaMay) & 81 & 14 & 508 & - \\
\hline Cabra (@RociodeCabra) & 189 & 2 & 263 & - \\
\hline Málaga (@HDADMALAGA) & 1.935 & 569 & 598 & - \\
\hline Cádiz (@HdadCadiz) & 852 & 398 & 111 & - \\
\hline Puente Genil (@HdadRocioPGenil) & 538 & 23 & 198 & - \\
\hline Marbella (@HdRocioMarbella) & 213 & 238 & 227 & - \\
\hline Tocina (@RocioTocina) & 119 & 21 & 102 & - \\
\hline Utrera (@HdadUtreraRocio) & 30 & 47 & 203 & - \\
\hline Almería (@hdadrocioalm) & 1.926 & 194 & 735 & - \\
\hline Cerro del Águila (@Hrociodelcerro) & 2.930 & 2.243 & 138 & - \\
\hline
\end{tabular}




\begin{tabular}{|c|c|c|c|c|}
\hline Sevilla Sur (@RocioSevillaSur) & 3.024 & 352 & 264 & - \\
\hline Toledo (@hermandadR) & 9 & 11 & 81 & - \\
\hline $\begin{array}{l}\text { Las Cabezas de San Juan } \\
\text { (@RocioLasCabezas) }\end{array}$ & 2.739 & 46 & 489 & - \\
\hline Fuengirola (@HdadRocioFuengi) & 556 & 115 & 195 & - \\
\hline Santiponce (@HdadRocioSantip) & 2.608 & 155 & 426 & - \\
\hline Valencia (@HdadRocioVIc) & 36 & 5 & 31 & - \\
\hline Mairena del Alcor (@XXVRocioMairena) & 461 & 104 & 114 & - \\
\hline Carmona (@HdRocioCarmona) & 176 & 96 & 208 & - \\
\hline Sevilla-Macarena (@HRocio_Macarena) & 1.479 & 1.312 & 227 & - \\
\hline Málaga-La Caleta (@HdadRocioCaleta) & 5.854 & 1.700 & 518 & - \\
\hline Ronda (@HdadRocioRonda) & 1.639 & 26 & 128 & - \\
\hline Chucena (@hdadrociochucen) & 50 & 41 & 239 & 1 \\
\hline La Algaba (@Hdadrocioalgaba) & 329 & 78 & 1.584 & - \\
\hline Murcia (@HdadRocioMurcia) & 576 & 173 & 144 & - \\
\hline Tomares (@RociodeTomares) & 711 & 17 & 93 & 1 \\
\hline Arcos de la Frontera (@HdadRocioArcos) & 950 & 50 & 125 & - \\
\hline Estepona (@HRocioEstepona) & 904 & 24 & 156 & - \\
\hline Alicante (@Hdad_Alicante) & 724 & 21 & 410 & - \\
\hline Mairena del Aljarafe (@RocioMairenaAlj) & 771 & 185 & 401 & 1 \\
\hline San Fernando (@HdadRocioSanFer) & 955 & 40 & 96 & - \\
\hline Morón de la Frontera (@HdadRocioMoron) & 888 & 22 & 159 & - \\
\hline Pozuelo de Alarcón (@HadRocioPozuelo) & 1.365 & 114 & 62 & - \\
\hline Cornellá (@HdadCornella109) & 1.756 & 39 & 104 & - \\
\hline Chipiona (@hdadchipiona) & 691 & 513 & 962 & - \\
\hline Castrense (@hdadrociocastre) & 110 & 41 & 232 & - \\
\hline
\end{tabular}




\begin{tabular}{|l|c|c|c|c|} 
Albaida del Aljarafe (@HRocioAlbaida) & 2.000 & 421 & 72 & - \\
\hline Santa Fe (@HdRocioSantaFe) & 43 & 38 & 112 & - \\
\hline Torrejón de Ardoz (@HdadTorrejon) & 2.142 & 1.129 & 469 & - \\
\hline Salteras (@rociosalteras) & 975 & 250 & 299 & - \\
\hline Valencina de la Concepción (@Valencina117) & 799 & 69 & 213 & - \\
\hline El Viso del Alcor (@HdadRocioViso) & 384 & 160 & 42 & - \\
\hline
\end{tabular}

Tabla 3. Twitter en las hermandades rocieras. Fuente: elaboración propia.

Como puede verse en la anterior tabla, cada una de las filiales rocieras que usa Twitter ofrece unos resultados distintos en cuanto al manejo corporativo de sus cuentas. La Hermandad de Málaga-La Caleta es la asociación que más tuits publica hasta la fecha de este estudio (5.854 tuits), seguida por la Hermandad Matriz de Almonte (4.082 tuits), mientras que la que más 'Me gusta' ha pulsado es la hispalense del Cerro del Águila (2.243). En el lado contrario, la filial de Punta Umbría es la que tiene menos publicaciones en Twitter (5 tuits), tal vez por ser más reciente su incorporación a esta red social, en concreto en septiembre de 2017. Y, además, hay cinco hermandades (Bollullos de la Mitación, Madrid, Emigrantes, Rota y Ayamonte) que no han marcado ni un 'Me gusta', dándose el caso de que dichas cuentas están en desuso o sin actualización desde antes de junio de 2017.

En cuanto al número de seguidores, de acuerdo con la tabla 2, la Hermandad Matriz de Almonte, con 20.669 seguidores, y la Hermandad de Triana, con 14.149, son las corporaciones rocieras con un mayor seguimiento por parte de los usuarios de la red social Twitter, muy por encima de otras hermandades.

Por otro lado, en este análisis destacamos la insuficiente modernización de algunas de estas cuentas, así como el escaso uso de las listas, sólo en siete hermandades (Matriz de Almonte, Pilas, Rociana del Condado, Punta Umbría, Chucena, Tomares y Mairena del Aljarafe), mientras que el número de seguidos, presente en todas ellas, va en función de cada filial rociera, claramente según la voluntad del gestor de estas cuentas. 


\begin{tabular}{|c|c|}
\hline Canal & Número de videos \\
\hline Hermandad Matriz de Almonte & 19 \\
\hline Villamanrique de la Condesa & 1 \\
\hline La Palma del Condado & 13 \\
\hline Moguer & 3 \\
\hline Triana & 14 \\
\hline Coria del Río & 22 \\
\hline Huelva & 1 \\
\hline Gines & 4 \\
\hline Olivares & 17 \\
\hline La Puebla del Río & 9 \\
\hline Bollullos Par del Condado & 3 \\
\hline Valverde del Camino & 1 \\
\hline Espartinas & 11 \\
\hline Sanlúcar la Mayor & 12 \\
\hline Punta Umbría & 2 \\
\hline Palos de la Frontera & 19 \\
\hline Villanueva del Ariscal & 6 \\
\hline Lucena & 11 \\
\hline Los Palacios y Villafranca & 7 \\
\hline Bormujos & 9 \\
\hline Lebrija & 2 \\
\hline Alcalá de Guadaira & 1 \\
\hline Cerro del Águila & 27 \\
\hline Sevilla Sur & 1 \\
\hline Almensilla & 7 \\
\hline
\end{tabular}




\begin{tabular}{|l|c|}
\hline Las Cabezas de San Juan & 4 \\
\hline Fuengirola & 9 \\
\hline Ceuta & 23 \\
\hline Valencia & 11 \\
\hline Mairena del Alcor & 16 \\
\hline Málaga-La Caleta & 6 \\
\hline Murcia & 4 \\
\hline Alcalá de Henares & 5 \\
\hline Guadix & 13 \\
\hline Palomares del Río & 47 \\
\hline Pozuelo de Alarcón & 1 \\
\hline Torrejón de Ardoz & 6 \\
\hline Salteras & 19 \\
\hline La Carolina & 3 \\
\hline El Viso del Alcor & \\
\hline
\end{tabular}

Tabla 4. YouTube en las hermandades rocieras. Fuente: elaboración propia.

En primer lugar, y de acuerdo con la tabla 2, más de la mitad de las cuentas de YouTube tienen una actualización escasa o nula, lo que creemos que afecta también a la suscripción y al interés del público por seguir a estos perfiles. En nuestro estudio, la Hermandad Matriz de Almonte es nuevamente la que tiene más suscriptores, en concreto 663, una cifra que es muy inferior a la registrada en las restantes redes sociales. Esta hermandad y la filial de Coria del Río, por ejemplo, se han unido en los últimos meses a esta red social y son de las más activas a fecha de octubre de 2018, si bien la filial con un mayor número de vídeos es Pozuelo de Alarcón, con 47.

Paradójicamente, según los resultados de la tabla 4, la segunda hermandad con más suscriptores, Huelva, con 179, es una de las que tiene menos vídeos subidos (sólo uno), 
mientras que la filial de Olivares, sin ningún suscriptor, tiene 17 vídeos. Hay otras dos hermandades, Lebrija y Alcalá de Guadaira, que tampoco cuentan con suscriptores y que, además, tienen pocos vídeos subidos ( 2 y 1 , respectivamente), estando todos estos perfiles no actualizados. Creemos nuevamente que en muchas ocasiones estas cuentas fueron creadas por anteriores juntas de gobierno y las actuales no publican nuevos contenidos. Incluso podrían encontrarse ejemplos de desconocimiento de la presencia de hermandades en alguna red social de las que se encuentran en desuso.

En resumen, como podemos ver en el caso de YouTube, los resultados de seguidores (suscriptores) y de publicaciones (vídeos), son los más bajos de todo el análisis efectuado en las cuatro redes sociales examinadas.

\begin{tabular}{|l|c|c|}
\hline \multicolumn{1}{|c|}{ Perfil } & Publicaciones & Seguidos \\
\hline Villamanrique de la Condesa & 1 & 1.400 \\
\hline La Palma del Condado & 63 & 561 \\
\hline Moguer & 53 & 28 \\
\hline Triana & 27 & 47 \\
\hline Umbrete & 7 & 1.364 \\
\hline Coria del Río & 275 & 54 \\
\hline Huelva & 164 & 232 \\
\hline Benacazón & 22 & 1.909 \\
\hline Gines & 314 & 9 \\
\hline Hinojos & 80 & 57 \\
\hline Lucena del Puerto & 3 & 121 \\
\hline Madrid & 7 & 19 \\
\hline Punta Umbría & 18 & 245 \\
\hline Puerto Real & 22 & 86 \\
\hline Emigrantes & 51 & \\
\hline
\end{tabular}




\begin{tabular}{|c|c|c|}
\hline Lucena & 32 & 102 \\
\hline Los Palacios y Villafranca & 98 & 20 \\
\hline Écija & 182 & 0 \\
\hline Lebrija & 27 & 30 \\
\hline Cabra & 5 & 184 \\
\hline Jaén & 14 & 20 \\
\hline Tocina & 6 & 165 \\
\hline Utrera & 17 & 370 \\
\hline Almería & 99 & 286 \\
\hline Sevilla Sur & 401 & 115 \\
\hline Fuengirola & 25 & 108 \\
\hline Ceuta & 156 & 2.010 \\
\hline Osuna & 3 & 221 \\
\hline Santiponce & 1.212 & 830 \\
\hline Sevilla-Macarena & 87 & 90 \\
\hline Málaga-La Caleta & 251 & 243 \\
\hline Alcalá la Real & 25 & 51 \\
\hline Chucena & 333 & 809 \\
\hline La Algaba & 39 & 1.098 \\
\hline Tomares & 41 & 57 \\
\hline Arcos de la Frontera & 27 & 699 \\
\hline Estepona & 300 & 271 \\
\hline Mairena del Aljarafe & 18 & 173 \\
\hline San Fernando & 37 & 330 \\
\hline Palomares del Río & 49 & 59 \\
\hline Morón de la Frontera & 90 & 62 \\
\hline
\end{tabular}




\begin{tabular}{|l|c|c|}
\hline San Antonio de Portmany & 29 & 134 \\
\hline Chipiona & 13 & 61 \\
\hline Santa Fe & 30 & 34 \\
\hline Torrejón de Ardoz & 274 & 204 \\
\hline El Viso del Alcor & 161 & 151 \\
\hline
\end{tabular}

Tabla 5. Instagram en las hermandades rocieras. Fuente: elaboración propia.

Sin duda, Instagram es una red social que se consolida actualmente, y las hermandades rocieras van apostando cada vez más por su uso. Tal es así que muchas de ellas se han incorporado a la red social en los últimos tiempos. La Hermandad de Santiponce es la que hace un mayor empleo de esta red social, con 1.212 publicaciones, no siendo precisamente la que más seguidores tiene -que son Huelva y Triana, con 7.088 y 1.528 seguidores, respectivamente-, mientras que la filial de Villamanrique de la Condesa, en el otro extremo, sólo tiene una publicación.

Al igual que ocurre en Twitter, el número de seguidos de esta red social depende del criterio de seguimiento establecido por cada hermandad, siendo la de Ceuta la que a más personas y organizaciones sigue, en concreto a 2.010, una cifra más numerosa que sus 981 seguidores. Como decimos, es cada hermandad la que decide, según el criterio de la persona encargada de gestionar la cuenta, qué y cuándo publicar y a quién seguir. Entre todos los ejemplos posibles, a este respecto, se da incluso la circunstancia de que la Hermandad de Écija, con casi 200 publicaciones y 915 seguidores, no sigue a ninguno de ellos.

En resumen, como hemos podido observar al abordar nuestro objeto de estudio, se trata de la red social con perfiles más actualizados en el presente análisis, lo cual denota su vitalidad. Al estar de moda, creemos que próximamente más hermandades abrirán perfiles oficiales en Instagram para dar a conocer sus actividades.

\section{Discusión y conclusiones}


Las hermandades rocieras se han ido sumando poco a poco a la revolución de las redes sociales online, creando desde hace años diferentes perfiles en las ya veteranas plataformas Facebook, Twitter y YouTube, en la medida de lo posible y, en los últimos tiempos, incorporando otras más modernas como Instagram. Así, todas las hermandades cuentan con algún perfil en la red social de Mark Zuckerberg, si bien no siempre en las restantes.

Pero el hecho de tener presencia en las redes sociales no implica dar siempre un correcto uso de las mismas, pues en algunos casos su empleo es inexistente y su actualización es nula. Incluso hay corporaciones rocieras con varias cuentas, algunas en desuso. De esta forma muchas hermandades, por tener sus cuentas obsoletas o sin uso, no llegan a cumplir su función comunicativa, como hemos visto en muchos ejemplos de nuestro análisis.

En efecto, en primer lugar notamos la ausencia de una estrategia comunicativa clara en estas asociaciones religiosas, al entender que muchas de ellas no saben qué usos o posibilidades tienen las redes sociales y cómo y cuándo deben realmente utilizarlas. A partir de este punto, aparecen otros aspectos que tienen que ver con los gestores de estas cuentas, especialmente con su formación y con el grado de interés que demuestran para informar y potenciar la participación, la viralidad y la popularidad de sus mensajes, entradas, vídeos y fotografías. La presencia de las hermandades en las redes sociales analizadas, en cuanto al número de tuits, vídeos o publicaciones, ofrece interesantes datos que confirman esto. El número de seguidores, además, puede verse condicionado igualmente por esta situación.

Por ello, primeramente las hermandades rocieras deberían actualizar y/o crear sus páginas webs y/o blogs, incorporando hipervínculos a sus redes sociales, para facilitar el acceso de la información a los hermanos y a la ciudadanía en general. Una vez en los perfiles corporativos rocieros de las redes sociales, deberían mostrar en la cabecera o en el espacio oportuno la referencia a la página web de la hermandad, indicando que son cuentas oficiales, ofreciendo la información precisa y trabajando con profesionalidad la información transmitida a los hermanos y seguidores -mejor si es propia y actualizada- para obtener una mayor retroalimentación. Así podrán pasar a ser hermandades 3.0, con el objetivo último de atender y dialogar con sus hermanos y seguidores rocieros.

En conclusión, como ejemplo de asociaciones religiosas que utilizan las redes sociales para comunicar, pese a los esfuerzos realizados por las hermandades del Rocío, creemos que todavía tienen mucho que recorrer y mejorar con su presencia y con el uso de las redes 
sociales y, especialmente, bastante que aprovechar de las posibilidades que éstas ofrecen para lograr poner en contacto a estas organizaciones con sus seguidores. En base a estos resultados, por útimo, esta investigación podría dar lugar a otro trabajo futuro en el que se tenga en cuenta cómo es la relación de estos públicos con las hermandades.

Notas

(1) El 16 de enero de 2018 la junta de gobierno de la Hermandad Matriz de Almonte admitió a las dos últimas hermandades filiales hasta la fecha: San Sebastián de los Reyes y El Viso del Alcor.

(2) Hechos de los Apóstoles 1:8. Versión de Evaristo Martín Nieto.

\section{Bibliografía}

Barnett, G. A. (ed.). (2011). Encyclopedia of Social Networks. Davis: University of California.

Cancelo Sanmartín, M.; Rebeil Corella, M. A. y Gabino Campos, M. A. (2015). La comunicación institucional de la Iglesia Católica a través de las redes sociales. Revista Internacional de Relaciones Públicas, 9(V), 111-130. doi: http://dx.doi.org/10.5783/RIRP-9-2015-07$111-130$

Cartes Barroso, M. J. (2016). La necesidad de comunicar en las hermandades. El Precursor, boletín informativo de la Hermandad Sacramental de San Juan Bautista, 24(24), 16-18.

eMarketer (16/01/2018). Emarketer Unveils Latest Worldwide Social Users Estimates. Recuperado de https://www.emarketer.com/content/emarketer-unveils-latest-wordwidesocial-users-estimates?ecid=NL1001 
Fernández, S. (2008). Redes sociales. Fenómeno pasajero o reflejo del nuevo internauta. Telos: Cuadernos de Comunicación e Innovación, 76, 118-120.

Gandolfo, M. L. (2014). La comunicación institucional en las redes sociales digitales on line. Análisis de un caso. Questión, 41(1), 156-171.

Gómez y Méndez, J. M.; Méndez Muros, S. y García Estévez, N. (Eds.). (2015). Periodismo y Rocío. Colección Pliegos de Información, 31. Sevilla: Equipo de Investigación de Análisis y Técnica de la Información de la Universidad de Sevilla.

Herrero Curiel, E. (2013). Periodistas y redes sociales en España. Del 11 M al 15M (20042011). (Tesis doctoral). Universidad Carlos III de Madrid, Madrid. Recuperado de http://fcic.periodistes.cat/wp-content/uploads/2013/11/Estudi-Periodistas-y-redessociales-en-Espa\%C3\%B1a.pdf

IAB (2018). Estudio anual de redes sociales. Recuperado de https://iabspain.es/wpcontent/uploads/estudio-redes-sociales-2018 vreducida.pdf

La Porte, J. M. (2012). Comunicación institucional. En La Porte, J. M. (Ed.). Introducción a la comunicación institucional de la Iglesia (201-225). Madrid: Palabra.

O'Reilly, T. (30/09/2005). What is Web 2.0? Design Patterns and Business Models for the Next Generation of Software. O'Reilly Media. Recuperado de http://www.oreilly.com/pub/a/web2/archive/what-is-web-20.htm

Ortiz de Zárate Tercero, A. (2008). Manual de uso del blog en la empresa. Cómo prosperar en la sociedad de la conversación. Barcelona: Infonomia. Recuperado de http://www.infonomia.com/img/libros/pdf/BlogsEmpresa.pdf

Padilla Díaz de la Serna, S. (2007). Rocío. La explosión de la gran devoción del sur en el siglo $X X$. Córdoba: Almuzara.

Pew Research Center (2015). The Evolving Role of News on Twitter and Facebook. Recuperado de http://www.journalism.org/files/2015/07/Twitter-and-News-SurveyReport-FINAL2.pdf

Ryan, P. K. (2011). Social networking. Digital and information literacy. New York: Rosen Publishing. 
Van Dijck, J. (2013). The culture of connectivity: a critical history of social media. New York: Oxford University Press.

We Are Social y Hootsuite (2018). Digital in 2018. Southern Europe. Recuperado de https://es.slideshare.net/wearesocial/digital-in-2018-in-southern-e 